\title{
WR 35a: A new double-lined spectroscopic binary ${ }^{\star}$
}

\author{
R. Gamen ${ }^{1, \star \star}$, A. Collado ${ }^{2, \star \star \star}$, R. Barbá ${ }^{3, \star \star \star \star}$, A.-N. Chené ${ }^{4}$, and N. St-Louis ${ }^{5}$ \\ 1 Instituto de Astrofísica de La Plata, CCT La Plata-CONICET, Facultad de Ciencias Astronómicas y Geofísicas, \\ Universidad Nacional de La Plata, Paseo del Bosque S/N, (1900) La Plata, Argentina \\ e-mail: rgamen@fcaglp .unlp.edu .ar \\ 2 Instituto de Ciencias Astronómicas de la Tierra y del Espacio (ICATE), CONICET, Avda. España 1512 Sur, J5402DSP San Juan, \\ Argentina \\ 3 Departamento de Física, Universidad de La Serena, Av. Cisternas 1200 Norte, La Serena, Chile \\ ${ }^{4}$ Gemini Observatory, Northern Operations Center, 670 North A'ohoku Place, Hilo HI 96720, USA \\ 5 Département de physique, Université de Montréal, CP 6128, Succ. Centre-Ville, Montréal (QC) H3C 3J7, Canada
}

Received 4 December 2013 / Accepted 7 December 2013

\begin{abstract}
Aims. We present the first orbital solution for the Wolf-Rayet star, WR 35a, that was discovered by a spectroscopic monitoring of faint WN-type stars.

Methods. Spectral features of two different components were identified, and thus a method of disentangling the individual spectra of both components was applied. Radial velocities were determined for each component in the binary system.

Results. The orbital solution and component properties of the system were derived. We determined that WR 35a is composed of a WN6 star with a O8.5 V companion orbiting at a 41.90-day period.
\end{abstract}

Key words. binaries: spectroscopic - stars: individual: WR 35a - stars: Wolf-Rayet

\section{Introduction}

Multiplicity seems to be a widespread property of massive stars. There are many studies pointing in this direction: e.g., Sana et al. (2012) and Sana et al. (2013) and many references therein. The observational evidence leads to an intrinsic binary fraction greater than $50 \%$.

Taking this observational fact into account, we are conducting a systematic spectroscopic monitoring campaign of galactic massive stars using both high- (OWN; Barbá et al. 2010) and low-resolution observations (Collado et al. 2013). The latter, only for faint WN-type stars, has resulted in the discovery of three double-lined binary systems (SB2), namely WR 62a, WR 68a, and WR 35a. SB2s are very important astrophysical objects since minimum stellar masses are derived from their orbital solution. Thus, they strongly contribute to our understanding of the evolutionary processes involved in such kind of stars.

WR 35a (=2MASS J11002434-5959357; $V=13.92$ ) is a Wolf-Rayet star discovered by Shara et al. (1991) and classified as WN6. They were not able to detect absorption lines in the spectrum. No other analysis of this star was found in the available bibliography. In the following, we report the discovery of its binary nature, show the individual spectrum of each component, and provide the first orbital solution.

\footnotetext{
* Table 2 is available in electronic form at http://www . aanda.org $\star \star$ Visiting astronomer, Cerro Tololo Inter-American Observatory (Chile) and CASLEO (Argentina).

$\star \star \star$ Fellow of CONICET, and visiting astronomer, Cerro Tololo InterAmerican Observatory (Chile) and CASLEO (Argentina).

$\star \star \star \star$ Visiting astronomer, Cerro Tololo Inter-American Observatory (Chile).
}

\section{Observations and data analysis}

The spectra used in this work were acquired between 1998 and 2013 with the $2.15 \mathrm{~m}$ telescope at Complejo Astronómico El Leoncito (CASLEO) ${ }^{1}$, Argentina; with the $4 \mathrm{~m}$ telescope at Cerro Tololo Inter-American Observatory (CTIO), Chile; and with the $8.1 \mathrm{~m}$ telescope at Gemini South Observatory ${ }^{2}$, Chile. See Table 1 for a summary of the observations ${ }^{3}$. In all cases, comparison lamp spectra of $\mathrm{He}-\mathrm{Ne}-\mathrm{Ar}$ ( or $\mathrm{Cu}-\mathrm{Ar}$ ) were observed immediately after or before, at the same telescope position as the stellar exposures. All spectra were processed with $\mathrm{IRAF}^{4}$ routines. The GMOs package was employed with Gemini data.

\subsection{Spectral disentangling}

We applied an iterative method for disentangling the individual spectra and computing the radial velocities (RVs) of both components in spectroscopic binaries developed by González \& Levato (2006). This technique consists in using the spectrum of one component to calculate the other. We used only our Gemini spectra since they constitute a homogeneous dataset,

\footnotetext{
1 Operated under agreement among CONICET and the Universities of La Plata, Córdoba, and San Juan, Argentina.

2 Under Poor Weather proposals GS-2008B-Q-87 (PI: ANCh) and GS2012B-Q-94 (PI: RG).

3 Reduced spectra are available at the NOVA's Data Center via http://nova. conicet.gov. ar.

4 IRAF is distributed by the National Optical Astronomy Observatories, which are operated by the Association of Universities for Research in Astronomy, Inc., under cooperative agreement with the National Science Foundation.
} 
Table 1. Details of the observations.

\begin{tabular}{|c|c|c|c|c|c|c|c|c|}
\hline $\begin{array}{l}\text { Obs. run } \\
\text { UT }\end{array}$ & $n$ & $\begin{array}{c}\text { Sp. coverage } \\
{[\AA]}\end{array}$ & Observat. & $\begin{array}{c}\text { Telesc. } \\
{[\mathrm{m}]}\end{array}$ & Spectr. $^{a}$ & $\begin{array}{l}\text { Grating } \\
{\left[1 \mathrm{~mm}^{-1}\right]}\end{array}$ & $\begin{array}{c}\text { Dispersion } \\
{\left[\AA \mathrm{pix}^{-1}\right]}\end{array}$ & Resolution $^{b}$ \\
\hline Feb., 1998 & 1 & $3940-5600$ & CASLEO & 2.15 & REOSC & 600 & 1.63 & 1000 \\
\hline Apr., 2000 & 3 & $3940-5600$ & CASLEO & 2.15 & REOSC & 600 & 1.63 & 1000 \\
\hline Apr., 2001 & 1 & $3940-5600$ & CASLEO & 2.15 & REOSC & 600 & 1.63 & 1000 \\
\hline Mar.-Apr., 2007 & 5 & $3650-6700$ & CTIO & 4 & $\mathrm{R}-\mathrm{C}$ & 632 & 1.01 & 1300 \\
\hline Apr., 2008 & 1 & $3650-6700$ & CTIO & 4 & $\mathrm{R}-\mathrm{C}$ & 632 & 1.01 & 1300 \\
\hline Mar., 2009 & 1 & $4030-5590$ & CASLEO & 2.15 & REOSC & 600 & 1.63 & 1000 \\
\hline Mar.-Jun., 2009 & 3 & $4120-6930$ & Gemini & 8.1 & GMOS & B600 & 0.92 & 1500 \\
\hline May, 2009 & 1 & $4470-5920$ & Gemini & 8.1 & GMOS & B1200 & 0.48 & 3000 \\
\hline Apr., 2010 & 3 & $4040-5700$ & CASLEO & 2.15 & REOSC & 600 & 1.63 & 1000 \\
\hline Apr., 2011 & 2 & $3930-5600$ & CASLEO & 2.15 & REOSC & 600 & 1.63 & 1000 \\
\hline Jan.-Feb., 2013 & 7 & $3920-6730$ & Gemini & 8.1 & GMOS & B600 & 0.92 & 1400 \\
\hline May, 2013 & 1 & $4030-5590$ & CASLEO & 2.15 & REOSC & 600 & 1.63 & 1000 \\
\hline
\end{tabular}

Notes. $(n)$ : Number of spectra obtained per run. ${ }^{(a)}$ Details of the spectrographs can be found in the User's Manuals of the respective Observatories. (b) The spectral resolutions $(R=\lambda / \Delta \lambda)$ were measured using $\Delta \lambda$ as the FWHM of the calibration lamp emission lines.

which sample the orbital phases and present good signal-to-noise ratios very well.

The method needs initial values of RVs to shift the spectra to a common velocity and to average them accordingly. Thus, we measured the $\mathrm{He}$ II $\lambda 4686$ emission line to represent the WN orbital motion and the He I $\lambda 4471$ absorption line to depict the secondary motion. The first step consists in shifting the input spectra by the WN's RVs and averaging them. It is thus expected that the secondary spectral features were diluted along the averaged spectrum. Then, all of the composite spectra are subtracted by this almost-pure WN spectrum (RV accordingly corrected), and the remaining spectra should be the secondary ones. These templates, which represent the individual spectra of both components, are then subtracted to each composite spectrum (paying attention to the respective RVs). The respective, almost-pure, individual spectra are cross-correlated (using FXCOR/IRAF) with the templates, and new RV are determined. These RVs are used again to calculate the new templates and repeat the cross-correlations. After several iterations, the templates and the RVs converge, and no improvements are obtained. Then, we used those templates to measure the RVs of the remaining spectra in the sample.

To calculate the templates, the spectral ranges used for the cross-correlation were $\lambda \lambda 4660-4710$ for the WN component and $\lambda \lambda 4460-4485,4532-4550,4910-4930,5005-5020$, and 5400-5425 for the secondary component. The RVs of each emission line of the WN component were determined using an appropiate spectral range including the line.

\section{Results and discussion}

\subsection{The individual spectra of both components}

WR 35a presents a composite spectrum of absorption and emission lines, not noted by Shara et al. (1991). As proved below, the absorption lines move in opposite directions to the emission lines, so they belong to a secondary component. We therefore applied a disentangling method (see Sect. 2.1) to separate the individual spectra and classify them. Both spectra are shown in Fig. 1.

The WN spectrum was already classified as WN6 by Shara et al. (1991) and later reclassified as WN6h by Shara et al. (1999). We agree with the later classification.

Gemini/GMOS capability was invaluable for obtaining a high-quality spectrum of the secondary component by means of the disentangling process. This spectrum shows absorption lines that we identified as He I $\lambda 4026,4387,4471,4713$, 4921, 5015, and 5875, He II $\lambda 4200,4541,4686$, and 5411, C III $\lambda 4069$, and 4650, and more marginally Mg II $\lambda 4481$ and $\mathrm{N}$ III $\lambda \lambda 4511-15$. We classified the secondary as O8.5 V through a visual comparison of the disentangled spectrum with those of the atlas of spectral standards published by Sota et al. (2011) using the MGB code (Maíz Apellániz et al. 2012).

In the spectrum of WR $35 \mathrm{a}$, the interstellar diffuse bands at $4430 \AA, 4500 \AA, 4726 \AA, 4760 \AA$, and $4775 \AA$, are visible among others, which indicates that the star is very reddened.

\subsection{The orbital solution}

As is well known in binary systems with components of WolfRayet type, the emission lines present different orbital solutions. Thus, we measured the RVs of the N IV $\lambda 4058$, He II $\lambda 4541$, 4686, and 5411, N V $\lambda 4604$, and N III $\lambda 4640$ emission lines independently (shown in Table 2). We searched for periodicities in the RV variations, using the Marraco \& Muzzio (1980) algorithm, and obtained a 41.9-day periodicity for all the measured features.

We determined the orbital elements of each emission line by means of the FOTEL code (Hadrava 2004) and proved that the emission lines present a similar orbital motion. In all cases, the periodicity resulted in almost the same value, thus we assumed a mean $P=41.898$ days. The eccentricity was found to be almost negligible, so we adopted a value of zero in all calculations.

The orbital parameters with the period and eccentricity fixed are shown in Table 3, ordered by the bounding energy of each transition superior level ( $E_{\mathrm{B}}$; Striganov \& Sventitskii 1968). The orbital solutions are depicted in Figs. 2-7.

From Table 3, it seems that the He II $\lambda 4686$ emission line is phase-shifted with respect to the other emissions by $0.7-2$ days (or between 0.02 and 0.05 of phase). This is also reported in other WN+OB binary systems (see, e.g., Collado et al. 2013) and is related to the fact that its formation region is not spherically symmetric; e.g., it could have additional components in the wind-wind collision region. The different systemic velocities ( $V_{\mathrm{WR}}$ ), except that of the He II $\lambda 4686$, seem to be correlated to their respective bounding energies. This behavior is expected if the expanding $\mathrm{WN}$ atmosphere presents a negative temperature gradient outwards, and it is frequently observed in $\mathrm{WN}+\mathrm{OB}$ systems (see, e.g., Niemelä 1973; Gamen 2004). The emission lines 
R. Gamen et al.: WR 35a: A new double-lined spectroscopic binary

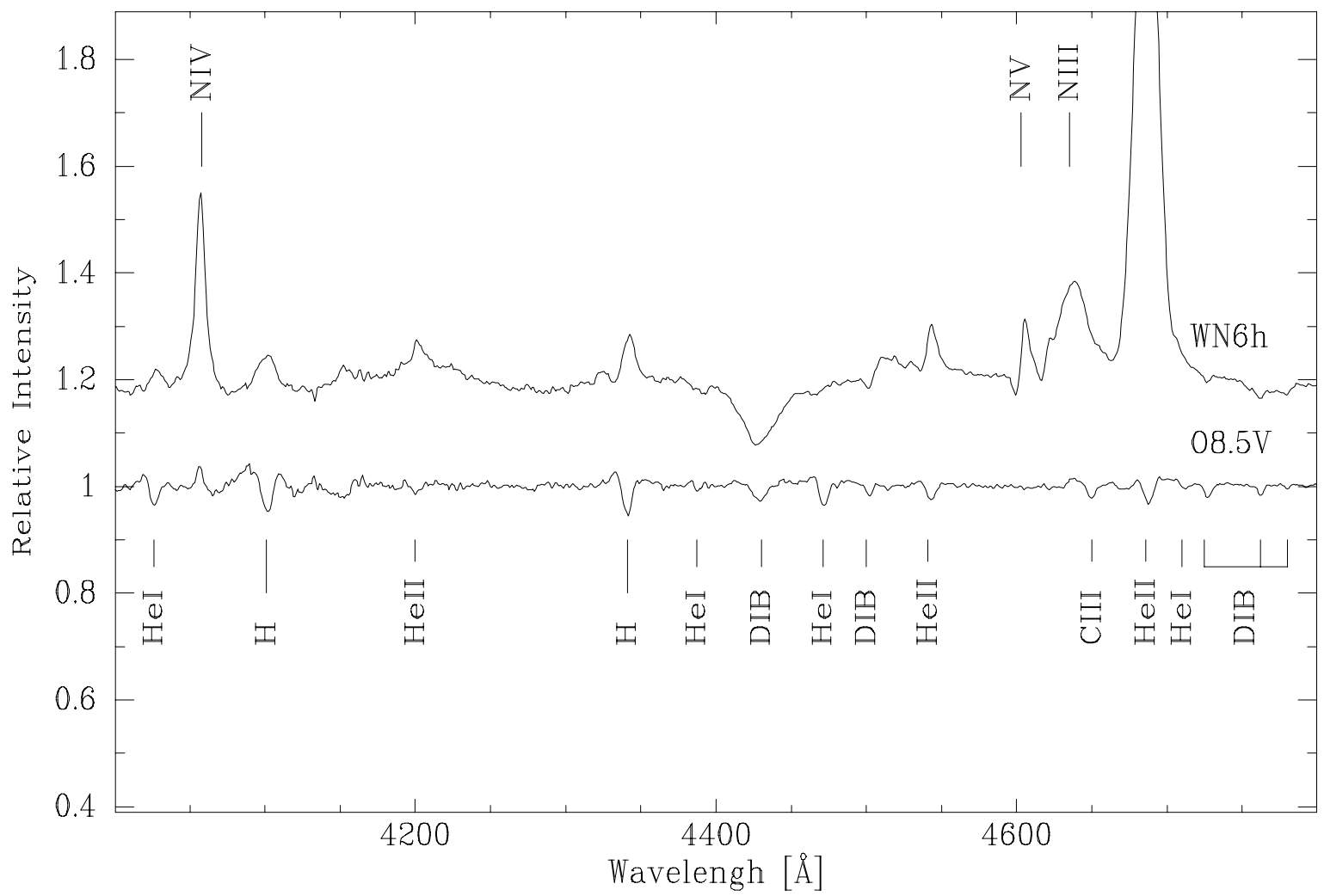

Fig. 1. Individual spectra of both components in WR 35a calculated by a disentangling method. The most important features are labeled below and above the respective spectra. Some diffuse interstellar bands (DIBs) are also identified.

Table 3. Circular orbital elements of WR 35a using different emission lines as representing the WR motion.

\begin{tabular}{lcccccc}
\hline \hline Parameter & N III $\lambda 4640$ & He II $\lambda 4686$ & N IV $\lambda 4058$ & He II $\lambda 5411$ & He II $\lambda 4541$ & N v $\lambda 4606$ \\
\hline$\lambda_{0}[\AA]$ & 4640 & 4685.68 & 4057.76 & 5411.52 & 4541.59 & 4603.73 \\
$E_{\mathrm{B}}[\mathrm{eV}]$ & 33.13 & 51.01 & 53.21 & 53.3 & 53.74 & 59.24 \\
$P[\mathrm{~d}]$ & & & $41.898 \pm 0.009$ & & \\
$T_{\mathrm{RV} \max }[\mathrm{HJD}-2455000]$ & $704.4 \pm 0.5$ & $703.7 \pm 0.4$ & $705.7 \pm 0.4$ & $705.0 \pm 0.4$ & $705.1 \pm 0.4$ & $704.4 \pm 0.4$ \\
$V_{\mathrm{WR}}\left[\mathrm{km} \mathrm{s}^{-1}\right]$ & -96 & 101 & -19 & 134 & 133 & 170 \\
$V_{\mathrm{O}}\left[\mathrm{km} \mathrm{s}^{-1}\right]$ & -15 & -15 & -15 & -15 & -15 & -15 \\
$K_{\mathrm{WR}}\left[\mathrm{km} \mathrm{s}^{-1}\right]$ & $34 \pm 11$ & $110 \pm 7$ & $119 \pm 9$ & $97 \pm 8$ & $70 \pm 10$ & $99 \pm 8$ \\
$K_{\mathrm{O}}\left[\mathrm{km} \mathrm{s}^{-1}\right]$ & 93 & 92 & 92 & 93 & 92 & 93 \\
$q\left[M_{\mathrm{O}} / M_{\mathrm{WR}}\right]$ & $0.37 \pm 0.06$ & $1.2 \pm 0.2$ & $1.3 \pm 0.2$ & $1.1 \pm 0.2$ & $0.8 \pm 0.2$ & $1.1 \pm 0.2$ \\
$a_{\mathrm{WR}} \sin i\left[R_{\odot}\right]$ & 28.1 & 91.0 & 98.8 & 80.5 & 58.1 & 81.9 \\
$a_{\mathrm{O}} \sin i\left[R_{\odot}\right]$ & 77.0 & 76.2 & 75.8 & 76.9 & 76.8 & 77.0 \\
$M_{\mathrm{WR}} \sin ^{3} i\left[M_{\odot}\right]$ & 6.5 & 16.3 & 17.7 & 14.6 & 10.7 & 14.9 \\
$M_{\mathrm{O}} \sin ^{3} i\left[M_{\odot}\right]$ & 2.4 & 19.5 & 23.0 & 15.3 & 8.1 & 15.8 \\
\hline
\end{tabular}

also have different amplitudes, so that the minimum masses derived are discrepant among them. However, it seems that the $\mathrm{O}$ component is more massive than the WN star. Then, the system is in an advanced evolutionary stage, where the originally more massive star has experienced high mass-loss rates and is currently the least massive one in the system.

\subsection{About the distance to WR $35 a$}

Bearing the above discussion in mind, we adopted the $\mathrm{N}$ V emission line as representing best the orbital motion of the WN component, and thus, the resulting minimum masses are $14.9 M_{\odot}$ for the WN6h and $15.8 M_{\odot}$ for the $08.5 \mathrm{~V}$ star. Assuming that the absolute mass of a $\mathrm{O} 8.5 \mathrm{~V}$ is $M=18.8 M_{\odot}$ (Martins et al. $2005)$, the orbital inclination is found to be about $i=71^{\circ}$, so the absolute mass of the WN should be $\sim 17.8 \mathrm{M}_{\odot}$.

Considering the evolutionary tracks of Ekström et al. (2012) in a very simplified scenario, a Wolf-Rayet star with this mass is compatible with $M_{\text {ini }} \sim 32 M_{\odot}$ of 5.4 Myr old (in the same sense, the $08.5 \mathrm{~V}$ star is compatible with $M_{\text {ini }} \sim 19 M_{\odot}$ of the same age). On the other hand, Collado et al. (2010) have discovered an early-type star in the surroundings of WR 35a. They relate this star to the 2MASS source J11002436-6000004 (=NOMAD1 0300-0240954 =USNO-B1 0300-0210713) and classify it as O9 III. We reclassify this spectrum as O9.5 II. The criteria for O8.5- to B0-type stars in Sota et al. (2011) are 


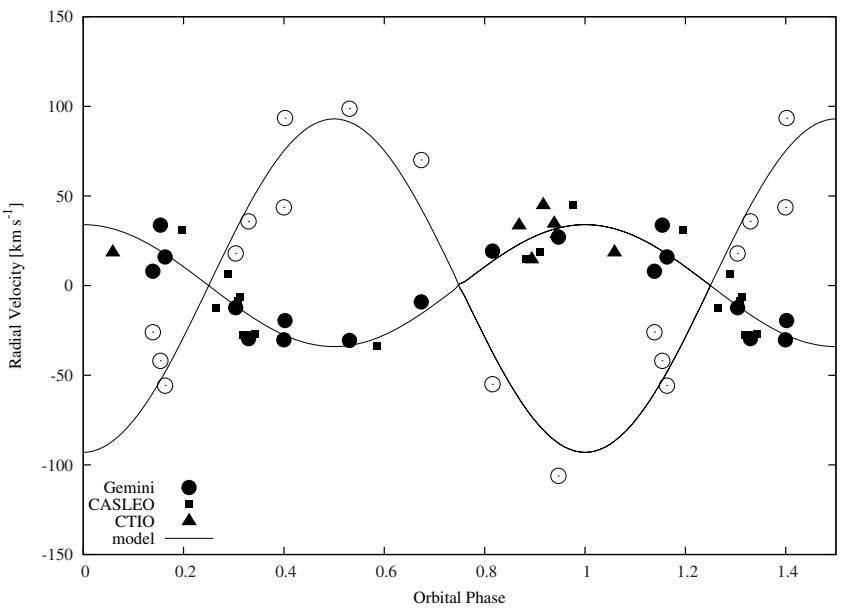

Fig. 2. Orbital solution of both components in WR 35a, using the $\mathrm{N}$ III $\lambda 4640$ as representing the WN motion.

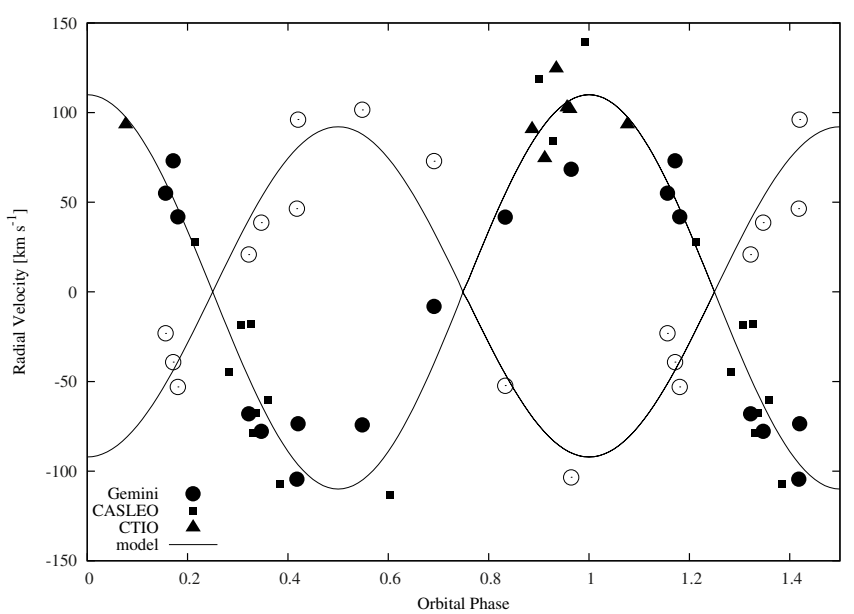

Fig. 3. Orbital solution of both components in WR 35a, using the He II $\lambda 4686$ as representing the WN motion.

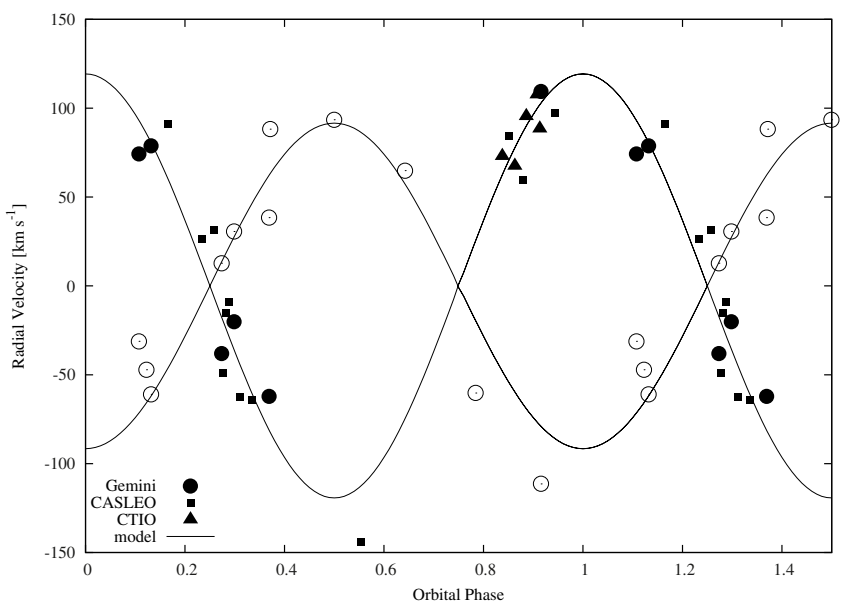

Fig. 4. Orbital solution of both components in WR 35a, using the N IV $\lambda 4058$ as representing the WN motion.

the He II $\lambda 4542 / \mathrm{He}$ I $\lambda 4388$ and He II $\lambda 4200 / \mathrm{He}$ I $\lambda 4144$ ratios, which in our case are much smaller than one. Also, the O9-O9.7 luminosity criteria He II $\lambda 4686 / \mathrm{He}$ I $\lambda 4713$ and Si IV $\lambda 4089 / \mathrm{He}$ I $\lambda 4026$ ratios indicate a class III from the former, but a class Iab from the latter. Thus, the final spectral type was

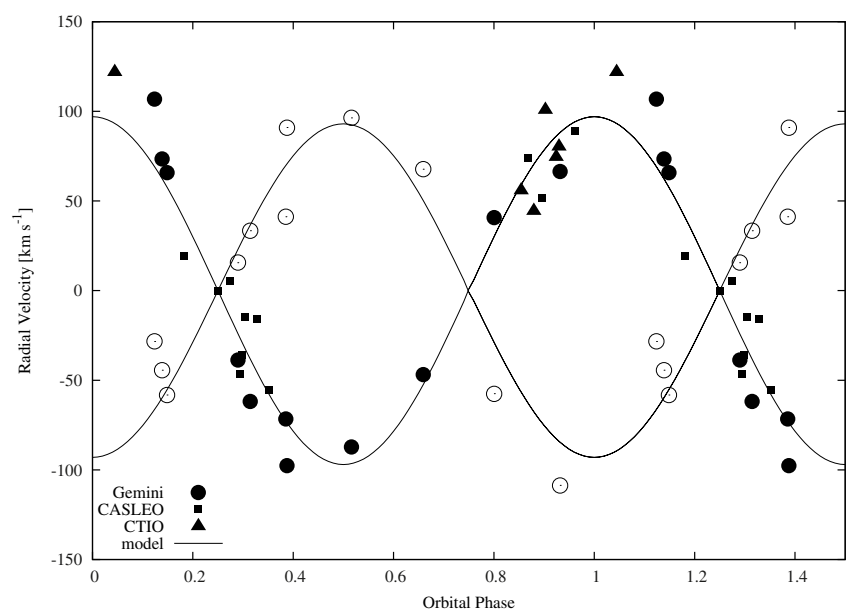

Fig. 5. Orbital solution of both components in WR 35a, using the He II $\lambda 5411$ as representing the WN motion.

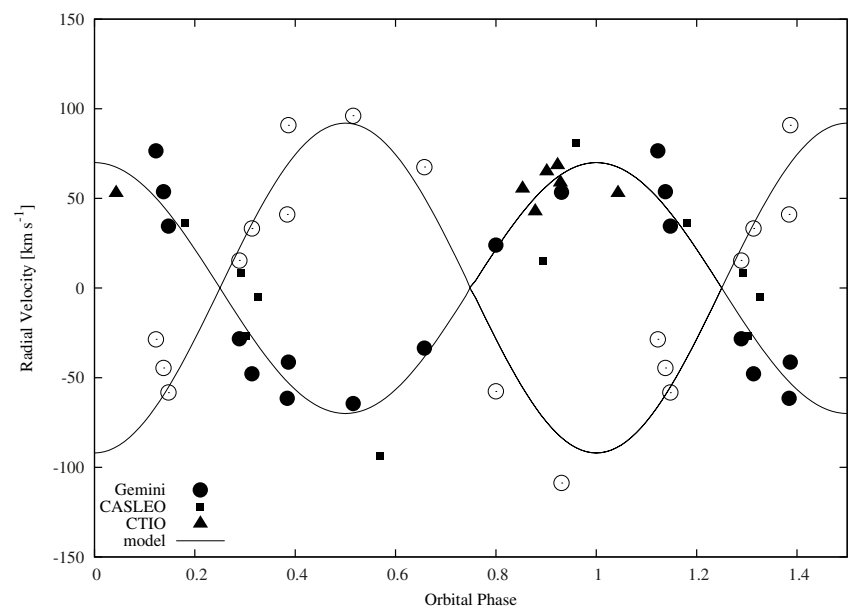

Fig. 6. Orbital solution of both components in WR 35a, using the He II $\lambda 4541$ as representing the WN motion.

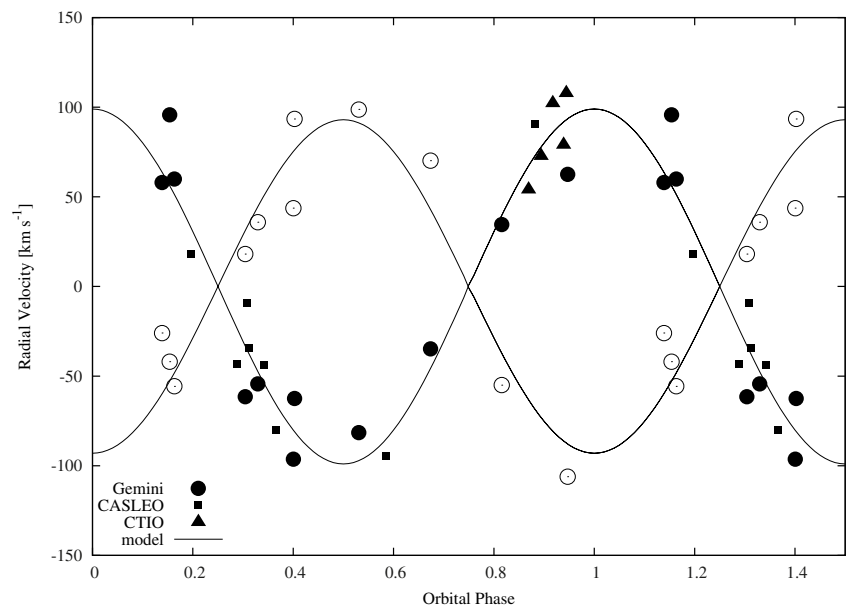

Fig. 7. Orbital solution of both components in WR 35a, using the $\mathrm{N}$ V $\lambda 4606$ as representing the WN motion.

decided by comparing the spectrum with the standard ones by means of the MGB code. Thus, this star could be assumed as having a mass of at least $21 M_{\odot}$ (Martins et al. 2005), hence adding up to at least three stars with masses above $19 M_{\odot}$ in a projected radius smaller than 0.50 arcmin. 
We could assume that these stars have a common origin, thus forming an $\mathrm{OB}$ association or open cluster. A preliminary spectro-photometric distance can be determined to the 2MASS source J11002436-6000004 using the $B$ and $V$ magnitudes provided by Zacharias et al. (2004) $)^{5}$ and assuming the absolute magnitude $M_{V}=-5.9$ (Walborn 1972), $B-V=-0.24$ (Wegner 1994), and the canonical ratio of total-to-selective extinction $R_{V}=3.1$. We obtained an extreme distance of $21 \mathrm{Kpc}$ using this $R_{V}$, but considering that this value seems to be very different towards the Carina arm (see Table 1 in Turner 2012), varying from the 3.1 to 5.5 , this estimation may be as small as $8.5 \mathrm{Kpc}$. This discrepancy should be resolved with further photometric and spectroscopic analysis, to determine the actual $R_{V}$ and thus find a more reliable distance. However, supposing equal interstellar extinction for both stars, the $m_{V}-M_{V} \sim 19$ of the O9.5 II star is

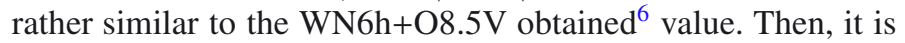
consistent with a common (unknown) distance.

\section{Conclusions}

We report the discovery of a new $\mathrm{WN}+\mathrm{O}$ binary system, where the $08.5 \mathrm{~V}$ component is probably more massive than the WN6h star, thus indicating an advanced evolutionary state. Minimum masses, $M_{\mathrm{WR}} \sin ^{3} i=15 M_{\odot}$ and $M_{\mathrm{O}} \sin ^{3} i=16 M_{\odot}$, were determined assuming the N v $\lambda 4606$ emission line represents the WN orbital motion, and the mean of some He I and He II absorption lines, the $\mathrm{O}$ component.

We discussed the distance and relationship of WR 35a and a O9.5 II neighboring star and proposed they may be at the same distance, hence forming an $\mathrm{OB}$ association or even an open cluster, which should be confirmed with follow-up observations.

Acknowledgements. Based on observations at the Gemini Observatory, which is operated by the Association of Universities for Research in Astronomy, Inc., under a cooperative agreement with the NSF on behalf of the Gemini partnership: the National Science Foundation (US), the National Research Council (Canada),
CONICYT (Chile), the Australian Research Council (Australia), Ministério da Ciência, Tecnologia e Inovação (Brazil), and Ministerio de Ciencia, Tecnología e Innovación Productiva (Argentina). We thank the directors and staff of CASLEO and CTIO for allowing us to use their facilities. We are grateful to the referee, Dr. Michael Shara, for his careful reading of the paper. R.H.B. acknowledges support from DIULS and FONDECYT Project 1120668. We also acknowledge Petr Hadrava for kindly allowing us to use the FOTEL code. This research has made use of the NASA's Astrophysics Data System and the SIMBAD database, operated at the CDS, Strasbourg, France.

\section{References}

Barbá, R. H., Gamen, R., Arias, J. I., et al. 2010, in Rev. Mex. Astron. Astrofis., Conf. Ser., 38, 30

Collado, A., Gamen, R., \& Barbá, R. 2010, Boletin de la Asociación Argentina de Astronomía, 53, 185

Collado, A., Gamen, R., \& Barbá, R. H. 2013, A\&A, 552, A22

Ekström, S., Georgy, C., Eggenberger, P., et al. 2012, A\&A, 537, A146

Gamen, R. 2004, Ph.D. Thesis, La Plata University, Argentina

González, J. F., \& Levato, H. 2006, A\&A, 448, 283

Hadrava, P. 2004, Publ. Astronomical Institute of the Czechoslovak Academy of Sciences, 92, 1

Maíz Apellániz, J., Pellerin, A., Barbá, R. H., et al. 2012, in Proceedings of a Scientific Meeting in Honor of Anthony F. J. Moffat, eds. L. Drissen, C. Rubert, N. St-Louis, \& A. F. J. Moffat, ASP Conf. Ser., 465, 484

Marraco, H., \& Muzzio, J. 1980, PASP, 92, 700

Martins, F., Schaerer, D., \& Hillier, D. J. 2005, A\&A, 436, 1049

Monet, D. G., Levine, S. E., Canzian, B., et al. 2003, AJ, 125, 984

Niemelä, V. S. 1973, PASP, 85, 220

Sana, H., de Mink, S. E., de Koter, A., et al. 2012, Science, 337, 444

Sana, H., de Koter, A., de Mink, S. E., et al. 2013, A\&A, 550, A107

Shara, M. M., Smith, L. F., Potter, M., \& Moffat, A. F. J. 1991, AJ, 102, 716

Shara, M. M., Moffat, A. F. J., Smith, L. F., et al. 1999, AJ, 118, 390

Sota, A., Maíz Apellániz, J., Walborn, N. R., et al. 2011, ApJS, 193, 24

Striganov, A., \& Sventitskii, N. 1968, Tables of Spectral Lines of Neutral and Ionized Atoms (New York: Plenum)

Turner, D. G. 2012, Ap\&SS, 337, 303

van der Hucht, K. A. 2001, New Astron. Rev., 45, 135

Walborn, N. R. 1972, AJ, 77, 312

Wegner, W. 1994, MNRAS, 270, 229

Zacharias, N., Monet, D. G., Levine, S. E., et al. 2004, in Am. Astron. Soc. Meet. 205 Abstr., BAAS, 36, 1418

Page 6 is available in the electronic edition of the journal at http: //wWw . aanda. org

\footnotetext{
5 NOMADS compiles the $B$ magnitudes from Monet et al. (2003) and the $V$ ones from the YB6 Catalog (USNO, unpublished).

6 Assuming $M_{V}=-4.4$ for the $08.5 \mathrm{~V}$ (Walborn 1972), and $M_{V}=$ -4.1 for the WN6 (van der Hucht 2001), $m_{V}-M_{V} \sim 18$ is obtained. If the $M_{V}=-7$ of a WN6h is chosen instead, $m_{V}-M_{V} \sim 20$ is obtained.
} 
A\&A 562, A13 (2014)

Table 2. Radial velocities measured in the spectra of WR 35a.

\begin{tabular}{|c|c|c|c|c|c|c|c|}
\hline HJD-2 400000 & N IV $\lambda 4058$ & He II $\lambda 4541$ & N v $\lambda 4606$ & $\mathrm{~N}$ III $\lambda 4640$ & He II $\lambda 4686$ & He II $\lambda 5411$ & abs. \\
\hline 50868.772 & -74 & -86 & -31 & -18 & -55 & & \\
\hline 51653.659 & 61 & -20 & & -12 & -9 & 29 & \\
\hline 51654.637 & 8 & 2 & 19 & -11 & -2 & 27 & \\
\hline 51655.655 & 6 & & -17 & & -48 & -12 & \\
\hline 52013.620 & 130 & 22 & & 34 & 143 & 95 & \\
\hline 54190.594 & 83 & 57 & 79 & 40 & 123 & 135 & \\
\hline 54191.647 & 77 & 44 & 98 & 21 & 107 & 124 & \\
\hline 54192.615 & 105 & 66 & 128 & 51 & 157 & 180 & \\
\hline 54193.520 & 118 & 70 & 104 & 41 & 135 & 154 & \\
\hline 54193.749 & 98 & 60 & 133 & 35 & 134 & 160 & \\
\hline 54575.641 & & 54 & & 25 & 126 & 201 & \\
\hline 54916.580 & 162 & 43 & 81 & 47 & 87 & 63 & \\
\hline 54900.621 & & 51 & 83 & 39 & 99 & 89 & -62 \\
\hline 54972.490 & & -38 & -33 & -11 & -17 & -39 & 92 \\
\hline 54978.473 & & -7 & 14 & 10 & 49 & 1 & 64 \\
\hline 54998.588 & & 81 & 145 & 53 & 130 & 121 & -48 \\
\hline 55296.522 & 97 & & & 3 & 14 & 43 & -32 \\
\hline 55297.524 & 102 & & 20 & 22 & 41 & 49 & -62 \\
\hline 55298.532 & 55 & & 29 & 9 & -20 & 8 & \\
\hline 55657.567 & 155 & & 154 & 31 & 178 & 118 & \\
\hline 55661.485 & 168 & 88 & & 61 & 198 & 133 & \\
\hline 56296.791 & 88 & 103 & 107 & 27 & 112 & 155 & \\
\hline 56297.824 & 93 & 61 & 109 & 35 & 99 & 114 & \\
\hline 56303.734 & -24 & -2 & -13 & 7 & -11 & 9 & 12 \\
\hline 56304.790 & -6 & -21 & -6 & -10 & -21 & -14 & 29 \\
\hline 56307.843 & -48 & -15 & -14 & 0 & -17 & -50 & 87 \\
\hline 56307.746 & & -35 & -48 & -11 & -48 & -24 & 37 \\
\hline 56330.656 & 123 & 80 & 111 & 46 & 125 & 114 & -113 \\
\hline 56429.595 & 21 & 16 & 54 & 7 & 41 & -3 & \\
\hline
\end{tabular}

